\title{
Feasibility of airway segmentation from three-dimensional rotational angiography
}

\author{
Sebastian Goreczny ${ }^{1,2}$, Alexander Haak ${ }^{2,3,4}$, Gareth John Morgan ${ }^{2,3}$, Jenny Zablah ${ }^{2,3}$ \\ ${ }^{1}$ Polish Mother's Memorial Hospital, Research Institute, Lodz, Poland \\ ${ }^{2}$ Children's Hospital of Colorado, Aurora, Colorado, United States \\ ${ }^{3}$ University of Colorado Hospital, Aurora, Colorado, United States \\ ${ }^{4}$ Philips Healthcare, Andover, Massachusetts, United States
}

\section{Introduction}

Stent implantation for vascular lesions, as with branch pulmonary artery stenosis in patients with congenital heart disease has become a standard procedure with proven long-term efficacy [1]. Compression of an airway by a stent placed in an adjacent branch pulmonary artery is a rare but recognized complication, especially in patients with a complex cardiac anatomy [2]. This potential complication highlights the importance of adequate visualization of the vessel-airway relationship, which may influence an intervention plan in the cardiac catheterization laboratory $[3,4]$.

Flexible bronchoscopy has been used to guide endovascular stenting decreasing the risk of catastrophic airway compression [5]. Other specialists prefer cross-section imaging such as magnetic resonance imaging or computed tomography (CT) of the chest to better assess the vessel/airway relationship prior to a procedure and interventional planning [6].

Over the last decade three-dimensional rotational angiography (3DRA) has become a useful tool to assess complex cardiac anatomy in the catheterization laboratory [7]. The incremental adoption of 3DRA in clinical practice has demonstrated the advantages in identifying these anatomical spatial relationships in patients with congenital heart disease. The technique described herein, displays this relationship by simultaneous airway and vascular segmentation and reconstruction from 3DRA using commercially available tools.

\section{The limitation}

Although 3DRA has been gaining wider acceptance, there are few reports exploring the potential for integrating airway and vascular structures with this imaging modality. Truong et al. [8] showed the feasibility of creating multiplanar reformat images of the airways after 3DRA but were not able to segment them or produce a $3 \mathrm{D}$ reconstruction. A few reports describe the use of 3DRA for 3D presentation of topological airway relationships using a vendor specific angiographic software package $[4,9]$.

\section{The solution}

Outside cardiology, tools have been developed to facilitate identification and segmentation of anatomic features using cone beam CT (XperCT, Philips Healthcare) in a multi-slice $3 \mathrm{D}$ view, to guide interventional procedures like biopsies and embolization [10]. The present study investigated the use of interventional radiology software tool utilizing 3DRA images to develop a protocol for airway segmentation and reconstruction during cardiac catheterization in patients with congenital heart defects. XperGuide (Philips Healthcare) is a segmentation tool which allows stepwise 3D segmentation of structures with a low contrast ratio and irregular boundaries [10]. We developed a method of using this software package to allow presentation of the airway and the airway-vessel (pulmonary artery) relationship (Fig. 1). On

Address for correspondence: Sebastian Goreczny, MD, PhD, Department of Cardiology, Polish Mother's Memorial Hospital, Research Institute, ul. Rzgowska 281/289, 93-338 Łódź, Poland, tel: +48 42271 14 78, fax: +48 422711470 ,

e-mail: sebastiangoreczny@yahoo.pl

Received: $26.10 .2020 \quad$ Accepted: 13.10 .2020 


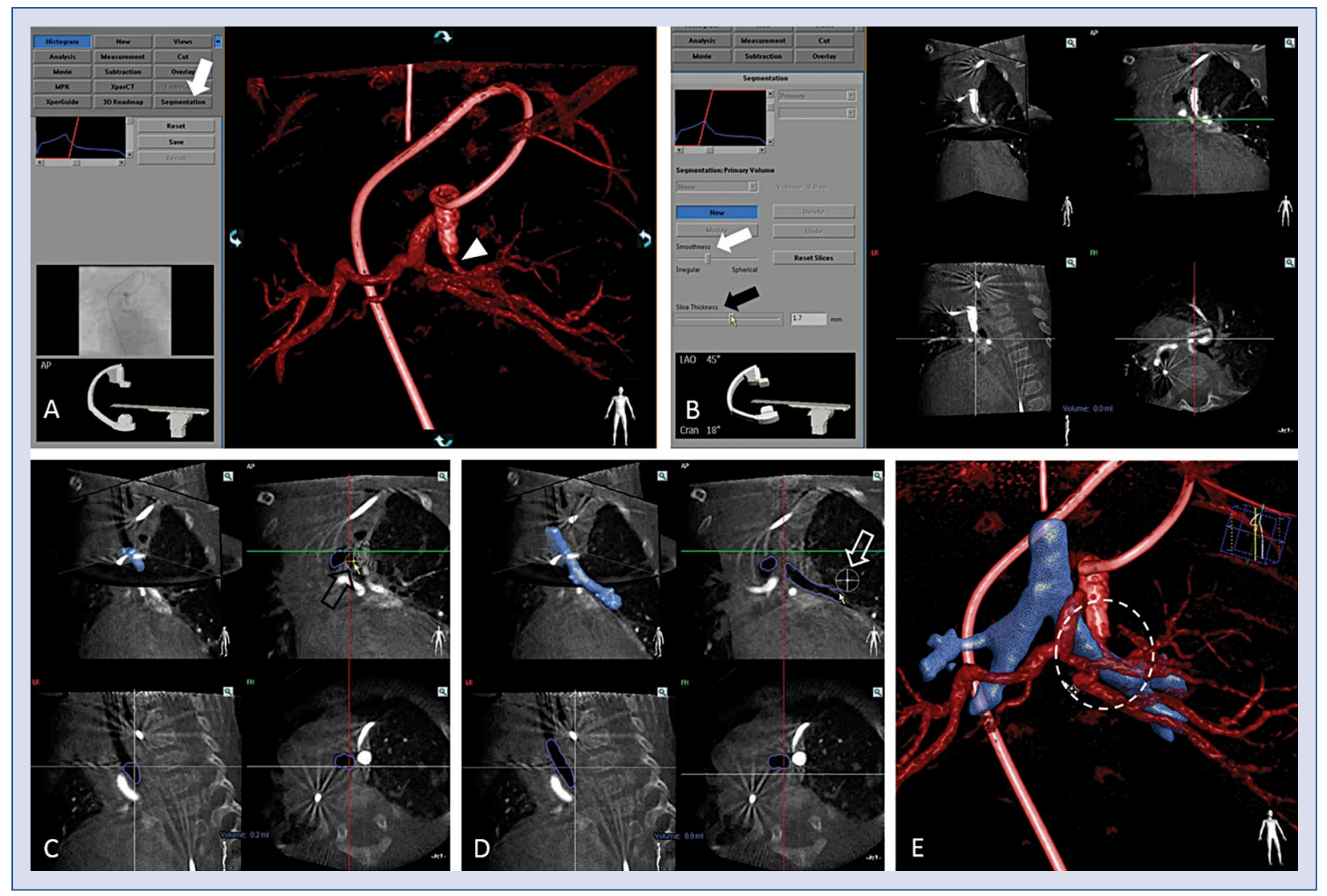

Figure 1. Step by step airway segmentation from three-dimensional rotational angiography (3DRA) in a patient after previous stent implantation to a major aorto-pulmonary collateral artery (MAPCA). A vascular reconstruction from 3DRA shows the narrow segment (white arrowhead) of the MAPCA distal to the previously implanted stent (A). Airway segmentation (white arrow) with XperGuide (Philips Healthcare) was performed for better visualization of the mechanism of the narrowing. Segmentation of the airway was conducted on three perpendicular planes of multiplanar reformats (B). At the beginning of the segmentation smoothness of the 3D airway and slice thickness of multiplanar reformats was selected according to operator preference. Manual growing of the airway was commenced at the level of the distal trachea and the proximal left main bronchus (black empty arrow) (C). Distal left main bronchus was corrected from outside (white empty arrow) (D). Finally, the vascular and airway 3D volumes were presented together clearly showing compression of the left main bronchus on distal segment of MAPCA (white dashed circle) (E).

a 3DRA data set the airway has a dark contrast (air) and can be manually segmented easily. Contrast filled pulmonary arteries can be automatically segmented and reconstructed with the potential for manual corrections where necessary. Both the vascular and airway structures can then be presented in the same virtual space allowing $3 \mathrm{D}$ visualization of the vessel and airway relationship (Fig. 2A-C). This complex can then be projected onto live fluoroscopy to guide cardiac catheterization.

\section{The validation}

Three-dimensional rotational angiography was performed in 23 patients for visualization of pulmonary arteries. Seventeen patients had biventricular circulations, whist the remaining 6 had single ventricle cardiac physiology at various stages of palliation. Figure 2D shows patient characteristics and procedural data. Airway segmentation was performed successfully in 21 patients $(21 / 23,91.3 \%)$. In 1 patient the isocenter of the imaging apparatus was set too anteriorly, resulting in only partial inclusion of the airway in the $3 \mathrm{D}$ volume. In the other patient, extensive artifacts from multiple, previously implanted vascular occlusion coils did not allow accurate visualization of the airways for segmentation.

The trachea was segmented in all patients (21), the left and right main bronchus in $21(100 \%)$ and $20(95 \%)$ patients, respectively (Fig. 2E). 


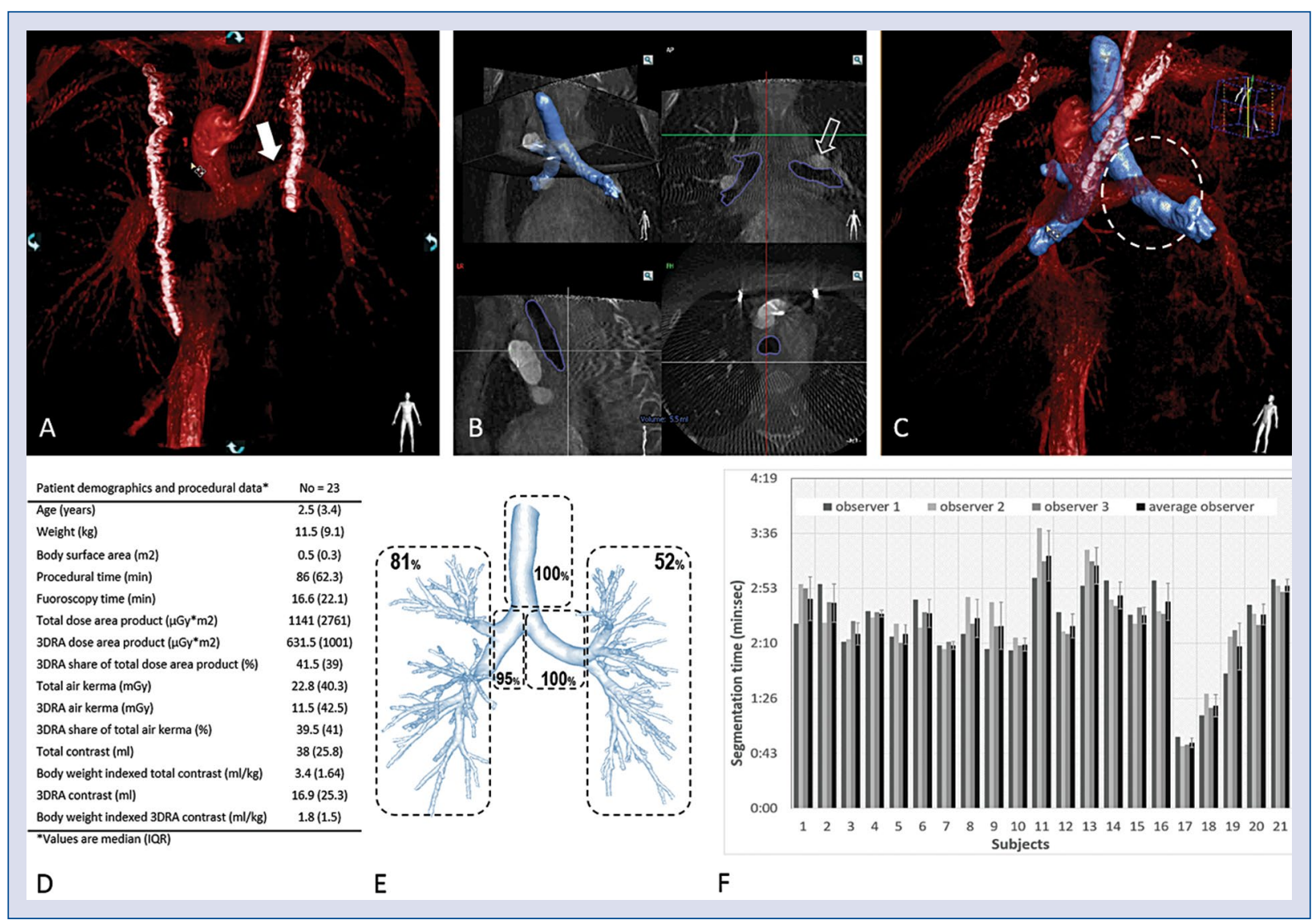

Figure 2. Example of airway segmentation (XperGuide, Philips Healthcare) from three-dimensional rotational angiography (3DRA) in patients after partial cavo-pulmonary connections (A-C). A raw reconstruction from 3DRA shows narrowing of the proximal left pulmonary artery (LPA, white arrow) (A). Already during airway segmentation on multiplanar reformats close relationship between the LPA (white empty arrow) and the left main bronchus (LMB) was noted (B). The vascular and airway 3D volumes, presented together, clearly reveal close relationships between the LPA and the LMB (white dashed circle) (C). Patient demographics and procedural data (D). Percentage of successful segmentation of particular airway segments $(\mathbf{E})$. Comparison of the segmentation times of the three independent observers (observer 1-3) and the average segmentation time (average observer) (F). The error bars represent the standard deviation of the segmentation time between the three observers. It can be noted that there was a good agreement in the segmentation times between the observers indicating that segmentation complexity and difficulties were experienced similarly.

Three patients were surreptitiously identified with airway anatomy variants or anomalies including 1 tracheal right upper bronchus, 1 patient diagnosed with right atrial isomerism based on the identification of bilateral morphological right bronchial anatomy, and 1 patient was noted to have major aortopulmonary collateral compression caused by the adjacent airway (Fig. 1). The median time for segmentation and production of an airway and vascular reconstruction was 2.4 (range $0.81-3.81$ ) min with no significant time difference between the three operators $(\mathrm{p}=0.9$; Fig. $2 \mathrm{~F})$.

\section{Conclusions}

This study shows the feasibility of quick and accurate airway segmentation from 3DRA. Segmentation of proximal airways was possible during a routine 3DRA acquisition of pulmonary arteries without any dedicated airway imaging protocol. As well as providing important incidental diagnoses in 3 patients, this rapid process provided valuable information for decision making and procedural guidance with overlay on live fluoroscopy. Further studies are warranted to explore clinical benefits of this technique of airway segmentation. 


\section{Conflict of interest: Alexander Haak is an em- ployee of Philips Healthcare.}

\section{References}

1. Ing FF, Khan A, Kobayashi D, et al. Pulmonary artery stents in the recent era: Immediate and intermediate follow-up. Catheter Cardiovasc Interv. 2014; 84(7): 1123-1130, doi: 10.1002/ ccd.25567, indexed in Pubmed: 24910458.

2. Moszura T, Mazurek-Kula A, Dryzek P, et al. Bronchial compression as adverse effect of left pulmonary artery stenting in a patient with hypoplastic left heart syndrome. Pediatr Cardiol. 2010; 31(4): 530-533, doi: 10.1007/s00246-009-9601-4, indexed in Pubmed: 19937008.

3. Moszura T, Dryzek P, Goreczny S, et al. A 10-year single-centre experience in percutaneous interventions for multi-stage treatment of hypoplastic left heart syndrome. Cardiol Young. 2014; 24(1): 54-63, doi: 10.1017/S104795111200220X, indexed in Pubmed: 23402359.

4. Krings GJ, van der Stelt F, Molenschot MMC, et al. Oval stenting in left pulmonary artery stenosis: a novel double balloon technique to prevent airway compression in single ventricle. EuroIntervention. 2020; 15(13): 1209-1215, doi: 10.4244/EIJD-18-01079, indexed in Pubmed: 30834894.

5. Ebrahim M, Hagood J, Moore J, et al. Bronchoscopic guidance of endovascular stenting limits airway compression. Catheter
Cardiovasc Interv. 2015; 85(5): 832-836, doi: 10.1002/ccd.25772, indexed in Pubmed: 25504498.

6. Grohmann J, Stiller B, Neumann E, et al. Bronchial compression following pulmonary artery stenting in single ventricle lesions: how to prevent, and how to decompress. Clin Res Cardiol. 2016; 105(4): 323-331, doi: 10.1007/s00392-015-0924-2, indexed in Pubmed: 26415706.

7. Góreczny S, Krings G, Hijazi ZM, et al. Pros, cons and future perspectives - three questions on three dimensional guidance for cardiac catheterization in congenital heart disease. Post Kardiol Interw. 2019; 15(3): 263-273, doi: 10.5114/aic.2019.87688, indexed in Pubmed: 31592250.

8. Truong UT, Fagan TE, Deterding R, et al. Use of rotational angiography in assessing relationship of the airway to vasculature during cardiac catheterization. Catheter Cardiovasc Interv. 2015; 86(6): 1068-1077, doi: 10.1002/ccd.26004, indexed in Pubmed: 26279410.

9. Borik S, Volodina S, Chaturvedi R, et al. Three-dimensional rotational angiography in the assessment of vascular and airway compression in children after a cavopulmonary anastomosis. Pediatr Cardiol. 2015; 36(5): 1083-1089, doi: 10.1007/s00246015-1130-8, indexed in Pubmed: 25762468.

10. Fu ZY, Feng Yu, Ma C, et al. Endovascular treatment of cavernous sinus dural arteriovenous fistulas via direct transorbital puncture using cone-beam computed tomography image guidance: report of 3 cases. World Neurosurg. 2019; 130: 306-312, doi: 10.1016/j.wneu.2019.07.002, indexed in Pubmed: 31299303. 